\title{
Estimates of Toxoplasmosis Incidence Based on Healthcare Claims Data, Germany, 2011-2016
}

\author{
Amrei Krings, Josephine Jacob, Frank Seeber, Uwe Pleyer, Jochen Walker, Klaus Stark, Hendrik Wilking
}

Toxoplasmosis is a zoonotic infection contracted through Toxoplasma gondii-contaminated food, soil, or water. Seroprevalence in Germany is high, but estimates of disease incidence are scarce. We investigated incidences for various toxoplasmosis manifestations using anonymized healthcare claims data from Germany for 2011-2016. Patients with a toxoplasmosis diagnosis during the annual observational period were considered incident. The estimated incidence was adjusted to the general population age/sex distribution. We estimated an annual average of 8,047 toxoplasmosis patients in Germany. The average incidence of non-pregnancyassociated toxoplasmosis patients was $9.6 / 100,000$ population. The incidence was highest in 2011, at 10.6 (95\% Cl 9.4-12.6)/100,000 population, and lowest in 2016 , at $8.0(95 \% \mathrm{Cl} 7.0-9.4) / 100,000$ population. The average incidence of toxoplasmosis during pregnancy was $40.3 / 100,000$ pregnancies. We demonstrate a substantial toxoplasmosis disease burden in Germany. Public health and food safety authorities should implement toxoplasmosis-specific prevention programs.

Toxoplasmosis is caused by infection with the pro1 tozoan parasite Toxoplasma gondii. Transmission of T. gondii can occur through food items and the environment. Main infection routes are the consumption of raw or undercooked meat or meat products containing $T$. gondii tissue cysts; ingestion of $T$. gondii oocysts through contaminated food items, such as fruits and vegetables; or ingestion of oocyst-contaminated soil or water (1). Most (80\%-90\%) infections in immune-competent persons are asymptomatic or manifest with mild influenza-like symptoms (2).

Author affiliations: Robert Koch Institute, Berlin, Germany (A. Krings, F. Seeber, K. Stark, H. Wilking); European Centre for Disease Prevention and Control, Stockholm, Sweden (A. Krings); InGef-Institute for Applied Health Research Berlin $\mathrm{GmbH}$, Berlin (J. Jacob, J. Walker); Charité Universitätsmedizin Berlin, Berlin (U. Pleyer)

DOI: https://doi.org/10.3201/eid2708.203740
However, infections in immunocompromised persons can cause severe disease manifestations and often occur as a reactivation of latent $T$. gondii infections (2). Disease manifestations can include meningoencephalitis, conjunctivitis, chorioretinitis, myocarditis, pneumonitis, and hepatitis. A primary infection with T. gondii during pregnancy can cause severe sequelae, known as congenital toxoplasmosis, for neonates and fetuses; these manifestations may include developmental delay, blindness, epilepsy, spontaneous abortion, and stillbirth $(3,4)$. Although T. gondii seroprevalence in several countries in Europe and the United States has slowly decreased over the past few decades (5-8), emerging collaborative and interdisciplinary One Health approaches may enable new prevention efforts that could substantially reduce the disease burden of toxoplasmosis.

In a systematic review, Torgerson et al. (9) estimated the global incidence and burden of disease for congenital toxoplasmosis as 190,100 (95\% CI 179,300-206,300) cases/year, which translates into an incidence rate of 1.5 cases/1,000 live births and a burden of disease of 1.2 million disease-adjusted life years (DALYs)/year. For Europe, an incidence rate of $0.5 / 1,000$ live births and a burden of disease of 2.8 DALYs/1,000 live births have been calculated (9). A meta-analysis reports a global incidence of acute toxoplasmosis during pregnancy as $1.1 \%$, ranging from $0.5 \%$ in the European region to $2.5 \%$ in Eastern Mediterranean region (10).

In Germany, T. gondii seroprevalence was previously found to range from $20 \%$ among patients $18-29$ years of age to $77 \%$ among patients $70-79$ years of age (11). The same study estimated 345 incident congenital toxoplasmosis cases per year (11). In contrast, routine surveillance data in Germany from 2009-2018 identified a minimum of 6 notified cases in 2014 and maximum of 23 cases in 2008. Medical doctors are required to report congenital forms of toxoplasmosis to the Germany national surveillance 
system, but not other forms of the disease or its diagnosis for patients infected with HIV or receiving organ transplants. We assumed substantial underreporting of diagnosis and underascertainment, which describes the potential absence of a diagnosis (12). Consequently, in Germany no data are available for the incidence of toxoplasmosis manifestations other than for congenital toxoplasmosis. However, having such estimates is essential to assess the disease burden and advise on appropriate and targeted prevention measures. Toxoplasmosis testing during pregnancy is not covered by the statutory health insurance in Germany; general screening of pregnant women has been shown to be cost-effective, but self-financed screening leads to selective testing of mostly women of higher educational status $(13,14)$. Although information about the risk for foodborne T. gondii infections during pregnancy is available to the public (15), there is no systematic monitoring of T. gondii in food products.

Disease surveillance of $T$. gondii is fragmented and unreliable; valid disease estimates could consequently inform and justify implementation of preventive measures. We aimed to estimate the annual incidence of different toxoplasmosis manifestations in Germany during 2012-2016. The method for using healthcare claims data in this study followed Lykins et al.'s approach for assessing toxoplasmosis estimates in the United States (16).

\section{Methods}

\section{Data and Definitions}

We obtained the study data from the anonymized healthcare claims database and provided by the Institute for Applied Health Research Berlin (InGef). Approximately 60 of the 123 statutory health insurance providers in Germany contribute to the database, which covers longitudinal data from $\approx 7$ million of the 83 million Germany residents. The characteristics of this dataset and its external validity have been described (17). The authors showed that, compared with the general population, the database population was slightly younger and includes a smaller proportion of East Germany inhabitants. However, rates of hospitalization and overall mortality and drug prescription rates were similar to those of the general population. The overall illness rates were slightly lower in the database population.

The study period covered 2011-2016. To estimate the annual toxoplasmosis incidence rates, we used inclusion criteria of continuous insurance with one of the statutory health insurance providers for $\geq 8$ quarters before the year analyzed and for all quarters of the year analyzed or until death (Figure 1). An exception was made for toxoplasmosis in mother and child, in which children needed to be insured since birth and pregnant women for $\geq 4$ quarters before and during the entire pregnancy to be included (Table 1).

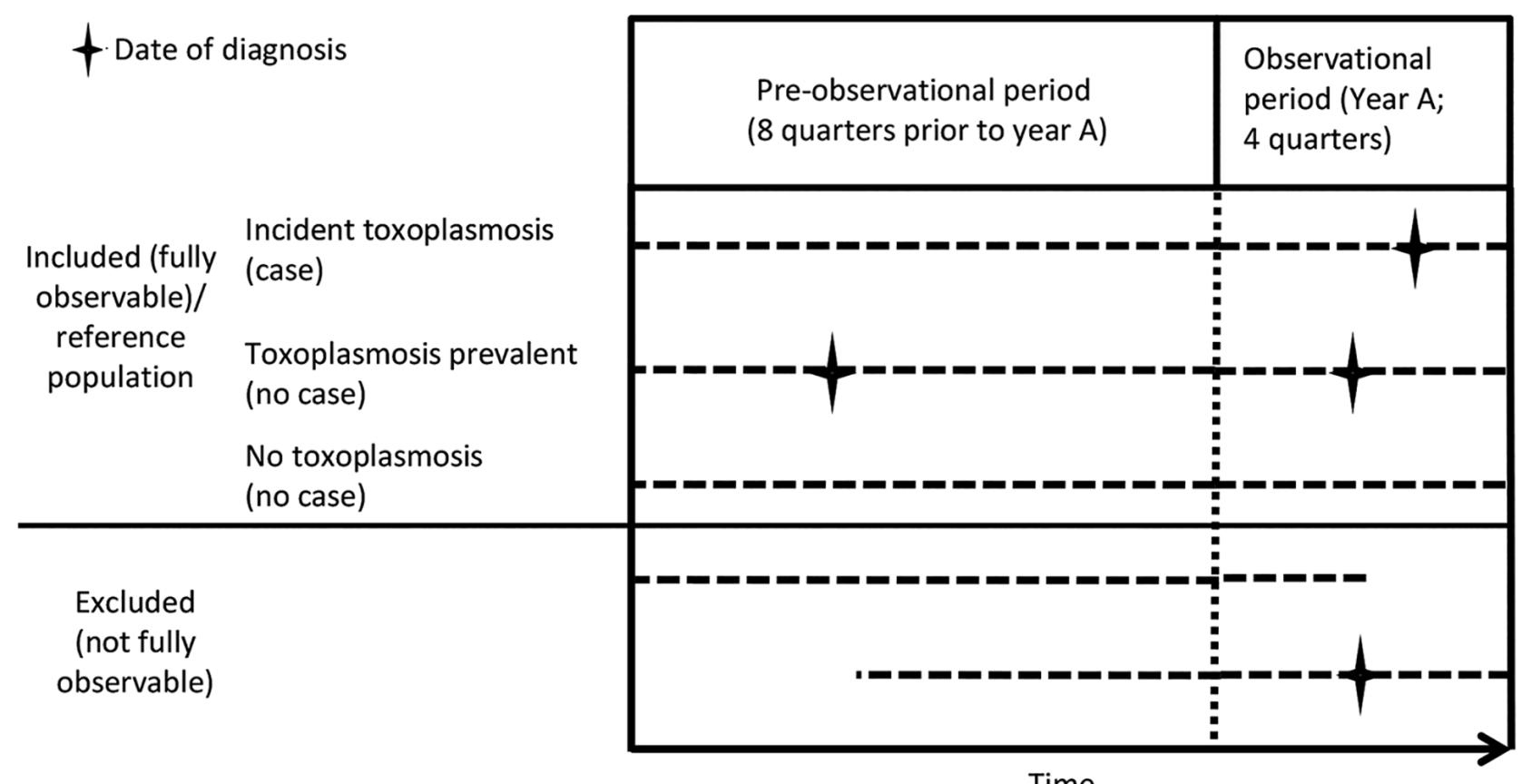

Time

Figure 1. Visualization of different inclusion and exclusion definitions for study of toxoplasmosis incidence based on healthcare claims data, Germany, 2011-2016. 
Table 1. Case definitions for toxoplasmosis disease manifestations used in analysis of healthcare claims, Germany*

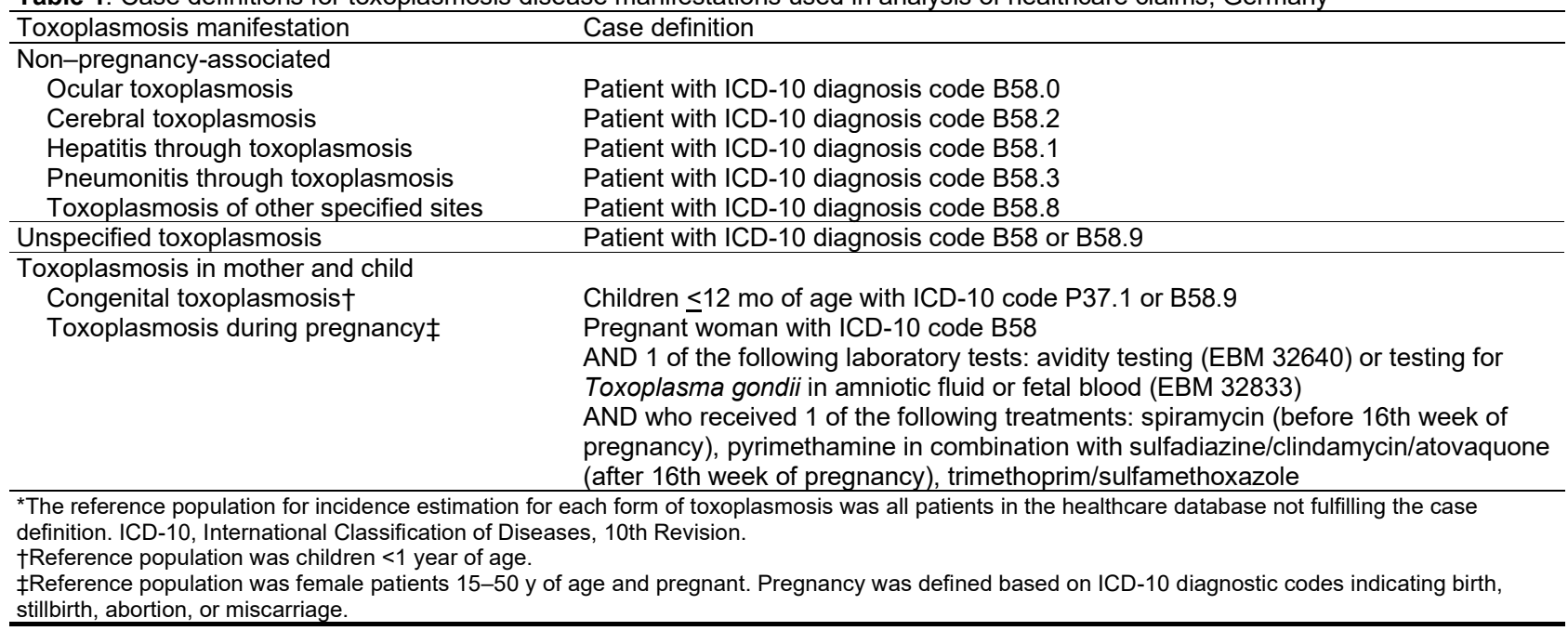

Case definitions for various toxoplasmosis manifestations were determined by diagnosis codes from the International Classification of Diseases, 10th Revision (ICD-10) (Table 1). The case definition for toxoplasmosis during pregnancy also includes toxoplasmosisspecific laboratory testing and therapy.

\section{Estimates of Incidence}

Toxoplasmosis patients are considered incident if they receive a diagnosis during the observational period but not in the preobservational period. For overall estimation of case numbers and incidence in Germany, we adjusted calculations in accordance with German Federal Statistical Office estimates for age, sex, and geographic distribution for each respective year. We calculated annual incidences for each toxoplasmosis manifestation separately and the average annual incidence as the arithmetic mean of the 6 yearly-determined incidence rates for 2011-2016. Patients identified in 2016, the most recent study period, were used for stratified analysis of geographic distribution of residence, sex, and age.

\section{Recurring Medical Claims of Toxoplasmosis}

To investigate potential toxoplasmosis relapse, we used recurring medical claims as an approximation. We defined any second medical toxoplasmosis claim as recurring if the patient had $\geq 2$ quarters without an existing ICD-10 code for toxoplasmosis; if the patient had a diagnosis of a different toxoplasmosis manifestation than that previously recorded; or if the patient record showed $\geq 2$ quarters without treatment while they still had the ICD-10 code. We differentiated rates of recurring medical claims per 100 person-years by relapse with the same or a different toxoplasmosis manifestation. Criteria for inclusion were a fully insured 4-quarter preobservational period before the first medical claim and continuous insurance throughout the 2012-2016 study period (Figure 2). We analyzed patients with recurring medical claims as proportions of all toxoplasmosis patients.

Underlying Conditions in Patients with $T$. gondii Infection For comparison, we used the scientifically reported and discussed underlying conditions provided in Lykins et al. (16) to analyze those conditions for Germany in this analysis (Appendix Table 1, https:// wwwnc.cdc.gov/EID/article/27/8/20-3740-App1. pdf). We defined toxoplasmosis cases as described previously (16) (Table 1). Toxoplasmosis-negatives were cases insured without any recorded episode of toxoplasmosis. Inclusion criteria were similar to those for the assessment of recurring medical claims (Figure 2); we excluded pregnant women and children $\leq 12$ months of age from this subanalysis. We matched the toxoplasmosis and reference groups 1:1 on the basis of age (by 5-year age groups), sex, and quarter of the diagnosis date of the underlying illness. Matching by quarter of diagnosis helped to avoid confounding due to seasonal variation of some underlying conditions. We calculated odds ratios for measure of association based on the frequencies of predefined conditions among the toxoplasmosis and reference group.

\section{Results}

\section{Study Population}

Of $\approx 7$ million persons insured during 2011-2016, we determined that 4.8-5.2 million were eligible for inclusion in our analysis per year. We found 2,625 


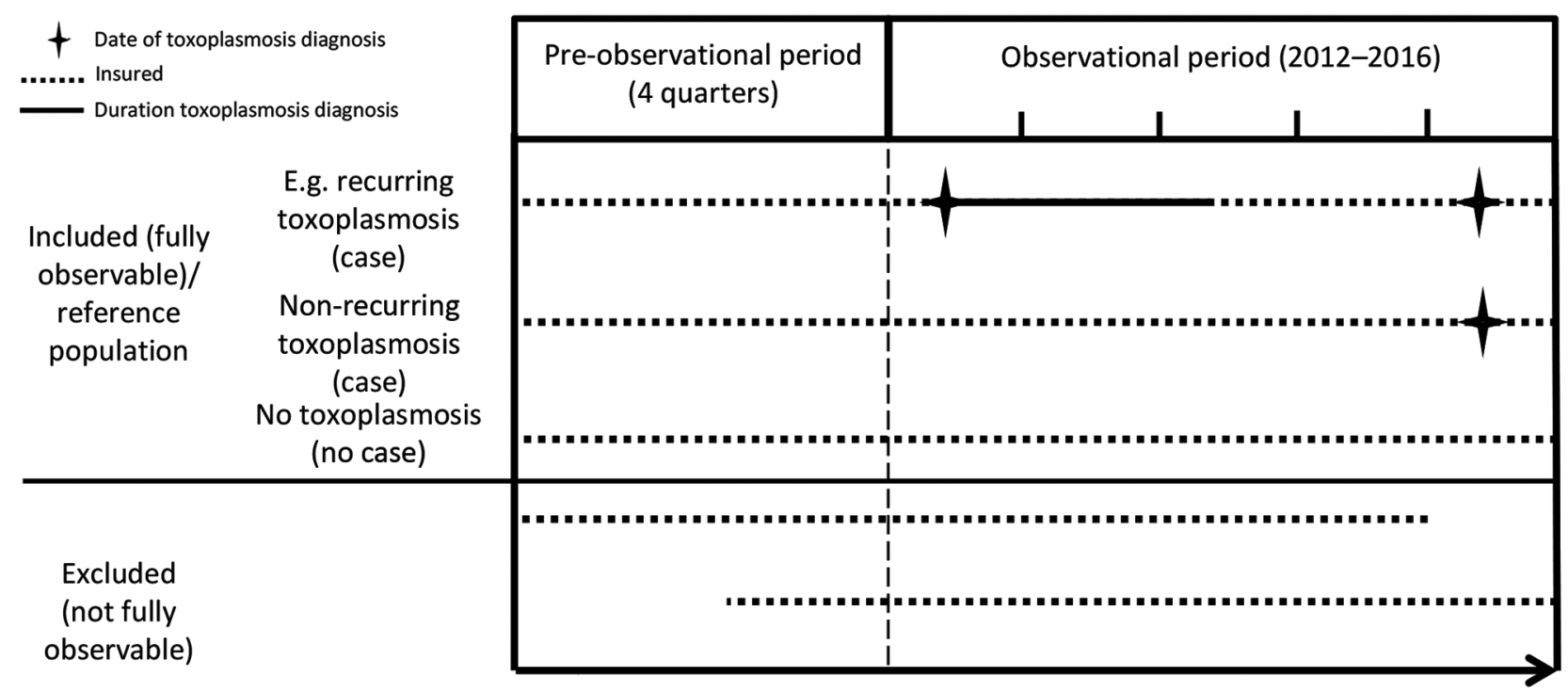

Figure 2. Visualization of different inclusion and exclusion definitions of recurring medical claims and identification of underlying conditions for study of toxoplasmosis incidence based on healthcare claims data, Germany, 2011-2016.

toxoplasmosis patients who met the case definitions in the database for these years, which is equivalent to 48,368 patients among $\approx 83$ million Germany residents. This total corresponds to an average annual case number of 8,061 patients. For 950/2,625 (36\%) of patients, a classification in one of the specific toxoplasmosis manifestations was possible; most patients had no specified toxoplasmosis manifestation.

\section{Temporal and Geographic Distribution}

Incidence for non-pregnancy-associated toxoplasmosis was as low as 8.0 (95\% CI 7.0-9.4)/100,000 population in 2016 and as high as 10.6 (95\% CI 9.4-12.6)/100,000 population in 2011. The average annual incidence of non-pregnancy-associated toxoplasmosis cases was $9.5 / 100,000$ population. Geographically, in 2016, the incidence of 4.5 (95\% CI 3.1-6.5)/100,000 population in Baden-Württemberg was significantly lower than the incidence of 12.5 (95\% CI 6.6-25.1)/100,000 population in Berlin and 9.1 (95\% CI: 6.7-12.2)/100,000 population in Lower Saxony (Figure 3).

\section{Ocular Toxoplasmosis}

We estimated an average annual case number of 1,601 cases of ocular toxoplasmosis and an average annual incidence of 2.0/100,000 population in Germany. The estimated incidence of ocular toxoplasmosis fluctuated between 1.5 (95\% CI 1.1- 2.3) patients/100,000 population in 2016 and 2.5 (95\% CI 2.0-3.8) patients/100,000 population in 2013 (Table 2). In 2016, the highest incidences of ocular toxoplasmosis were seen among women and in the 51-60-year age group (Table 3).

\section{Cerebral Toxoplasmosis}

The average annual number of cerebral toxoplasmosis patients in Germany was 142, and the average annual incidence was $0.18 / 100,000$ population. Incidence for cerebral toxoplasmosis was 0.1 cases $/ 100,000$ population in 2012, 2013, and 2014 and 0.3 cases/100,000 population in 2015 (Table 2). Because very few cases were recorded in the database, we were unable to stratify estimates for most sociodemographic characteristics (Table 4).

\section{Other Types and Nonspecified Types of Toxoplasmosis}

The average annual number of patients with toxoplasmosis manifestations at other sites, including those with hepatitis or pneumonitis from toxoplasmosis, was 752 patients. The average annual number of unspecified toxoplasmosis patients was 5,202 and the average annual incidence was $6.4 / 100,000$ population. In 2016, toxoplasmosis incidence was significantly higher among patients who were $21-40$ years of age compared with patients $<21$ years or $>40$ years of age $(\mathrm{p}<0.05)$ (Table 5$)$.

\section{Congenital Toxoplasmosis}

The average annual number of congenital toxoplasmosis patients was 8.2 and average annual incidence was $0.1 / 100,000$ population in Germany. Incidence estimates ranged from 0.05 (95\% CI 0.01-1.64)/100,000 pregnancies in 2012 to 0.14 (95\% CI 0.05-1.07)/100,000 in 2015 (Table 2). Our ability to estimate stratified incidence was limited because the number of cases found was low (Appendix Table 2). 


\section{Toxoplasmosis during Pregnancy}

The average annual number of patients with toxoplasmosis during pregnancy is 289 and average annual incidence is $40.3 / 100,000$ pregnancies in Germany. Incidence of toxoplasmosis during pregnancy fluctuated between 29.3 (95\% CI 16.7-56.4)/100,000 pregnancies in 2011 and 60.3 (95\% CI 33.0-107.5)/100,000 pregnancies in 2015 (Table 2).

Stratification by age group and geographic region was partly limited because of low case numbers. Therefore, estimation was only possible for women 31-40 years of age, who had an estimated incidence rate of 52.7 (95\% CI 24.1-138.1)/100,000 pregnancies. Stratification by region fluctuated between 34.4 (95\% CI 17.7-71.9)/100,000 pregnancies in the western region of Germany and 58.2 (95\% CI 17.2-177.9)/100,000 pregnancies in the northern region. No estimations are available for the middle and eastern regions (Table 6).

\section{Recurring Medical Claims of Toxoplasmosis}

Among all toxoplasmosis patients found in the dataset $(\mathrm{n}=2,776), 722(26 \%)$ had a recurring toxoplasmosis medical claim. The highest proportion of these $(250 / 574 ; 44 \%)$ was seen among patients who initially received a diagnosis of ocular toxoplasmosis. The rate of recurring medical claims (termed rate of claims in this study) among initial ocular toxoplasmosis patients was 19.8/100 person-years for any type of toxoplasmosis.
Among these patients, the rate of claims in patients with an additional episode of ocular toxoplasmosis was 16.8/100 person-years; for other manifestations, the rate of claims was 3.0/100 person-years.

\section{Underlying Conditions}

We calculated odds ratios for statistically significant underlying conditions found among toxoplasmosis cases, compared with matched controls based on 5-year age groups and sex (Table 7). Conditions that were significantly associated with toxoplasmosis were anxiety, epilepsy, lymphadenopathy, and thrombocytopenia, as well as vision loss or blindness (all $\mathrm{p}<0.05$ ). The conditions with $<5$ cases in the reference group but $>10$ cases in the toxoplasmosis group were HIV/AIDS; memory loss; and encephalitis, myelitis, or encephalomyelitis. We tested for other conditions that were not significantly associated with toxoplasmosis or affected $<5$ persons in either group (Appendix Table 1).

\section{Discussion}

Our study of toxoplasmosis incidence estimates and its manifestations, as determined from healthcare claims data, adds a valuable contribution to the evidence base. Most of the evidence on T. gondii infections and disease in Germany available to date is drawn either from serosurveys or from mandatory disease surveillance for congenital toxoplasmosis $(11,12)$. Therefore, our estimation

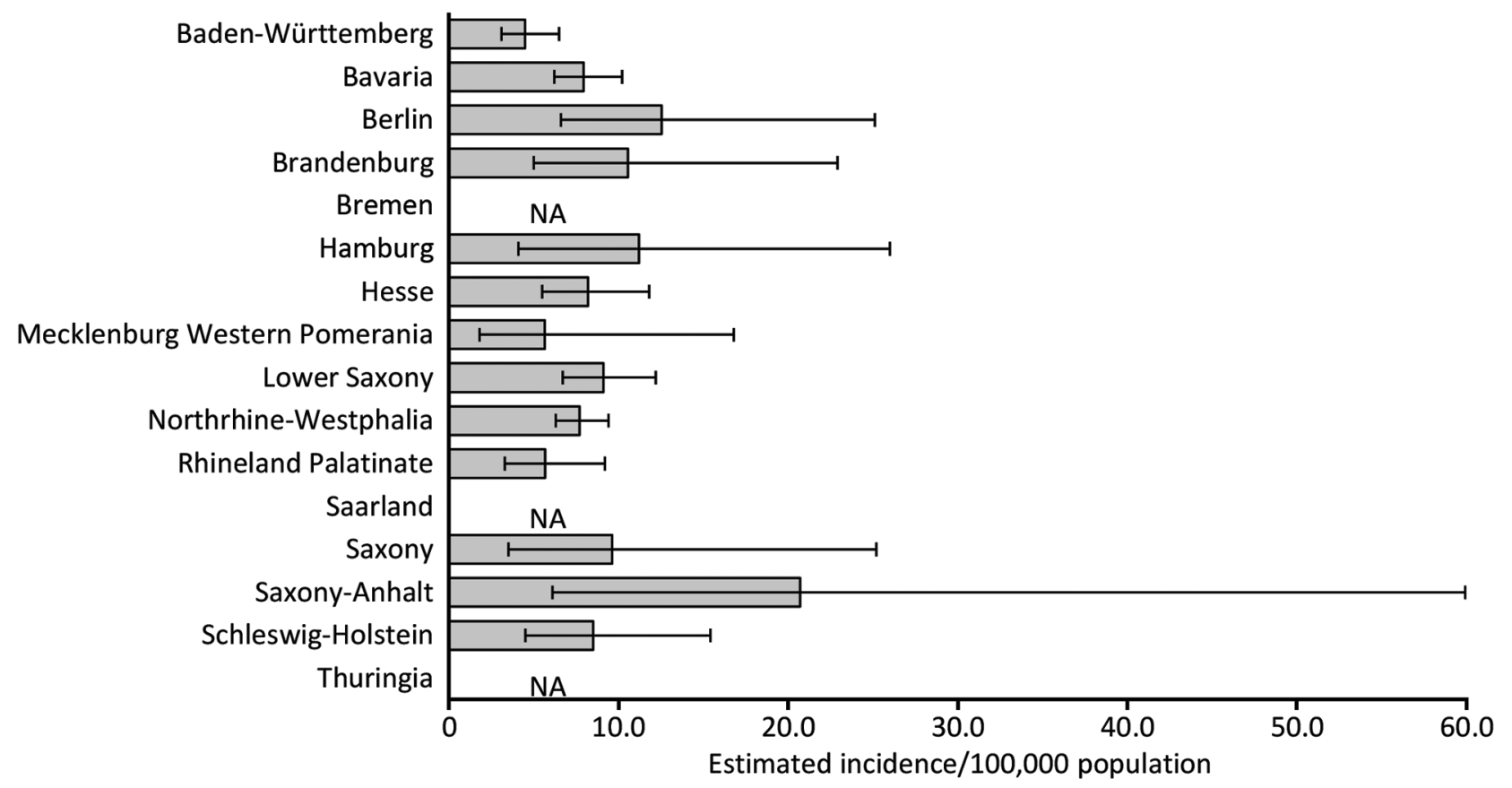

Figure 3. Toxoplasmosis disease incidence by federal state in Germany, 2016. Error bars indicate $95 \%$ Cls. No estimates were available for Bremen, Saarland, and Thüringen. NA, not available. 
Table 2. Estimated incidence of toxoplasmosis manifestations by year, Germany, 2011-2016

\begin{tabular}{|c|c|c|c|c|c|}
\hline Disease manifestation & Year & $\begin{array}{c}\text { No. patients in } \\
\text { database }\end{array}$ & $\begin{array}{c}\text { No. patients } \\
\text { identified }\end{array}$ & $\begin{array}{c}\text { Estimated no. patients } \\
(95 \% \mathrm{Cl})\end{array}$ & $\begin{array}{c}\text { Estimated cases } / 100,000 \\
\text { population }(95 \% \mathrm{Cl})\end{array}$ \\
\hline \multirow[t]{6}{*}{ Ocular toxoplasmosis } & 2011 & $4,705,497$ & 86 & $1,618(1,253-2,940)$ & $2.0(1.6-3.7)$ \\
\hline & 2012 & $4,751,579$ & 95 & 1,937 (1,522-3,189) & $2.4(1.9-4.0)$ \\
\hline & 2013 & $5,024,715$ & 111 & $2,017(1,623-3,029)$ & $2.5(2.0-3.8)$ \\
\hline & 2014 & $5,134,795$ & 74 & $1,359(1,015-2,241)$ & $1.7(1.3-2.8)$ \\
\hline & 2015 & $5,177,282$ & 76 & $1,452(1,035-2,342)$ & $1.8(1.3-2.9)$ \\
\hline & 2016 & $5,171,212$ & 75 & $1,224(941-1,931)$ & $1.5(1.1-2.3)$ \\
\hline \multirow[t]{6}{*}{ Cerebral toxoplasmosis } & 2011 & $4,705,497$ & 8 & $144(56-1,534)$ & $0.2(0.1-1.9)$ \\
\hline & 2012 & $4,751,579$ & 6 & $114(40-1,377)$ & $0.1(0.1-1.7)$ \\
\hline & 2013 & $5,024,715$ & 5 & $97(24-1,090)$ & $0.1(0.0-1.4)$ \\
\hline & 2014 & $5,134,795$ & 6 & $80(32-934)$ & $0.1(0.0-1.2)$ \\
\hline & 2015 & $5,177,282$ & 13 & $218(115-970)$ & $0.3(0.1-1.2)$ \\
\hline & 2016 & $5,171,212$ & 10 & $200(74-883)$ & $0.2(0.1-1.1)$ \\
\hline \multirow[t]{6}{*}{ Other types of toxoplasmosis $\dagger$} & 2011 & $4,705,497$ & 45 & 865 (NA) & NA \\
\hline & 2012 & $4,751,579$ & 40 & 715 (NA) & NA \\
\hline & 2013 & $5,024,715$ & 32 & 588 (NA) & NA \\
\hline & 2014 & $5,134,795$ & 58 & 912 (NA) & NA \\
\hline & 2015 & $5,177,282$ & 44 & 782 (NA) & NA \\
\hline & 2016 & $5,171,212$ & 43 & 651 (NA) & NA \\
\hline \multirow{6}{*}{ Nonspecified types of toxoplasmosis } & 2011 & $4,705,497$ & 306 & $5,866(5,093-7,406)$ & $7.3(6.3-9.2)$ \\
\hline & 2012 & $4,751,579$ & 283 & $5,503(4,703-6,981)$ & $6.8(5.8-8.7)$ \\
\hline & 2013 & $5,024,715$ & 265 & $5,177(4,483-6,389)$ & $6.4(5.6-7.9)$ \\
\hline & 2014 & $5,134,795$ & 237 & $4,277(3,686-5,327)$ & $5.3(4.5-6.6)$ \\
\hline & 2015 & $5,177,282$ & 309 & $5,821(5,070-6,936)$ & $7.1(6.2-8.4)$ \\
\hline & 2016 & $5,171,212$ & 242 & $4,569(3,862-5,628)$ & $5.5(4.7-6.8)$ \\
\hline \multirow[t]{6}{*}{ Congenital toxoplasmosis } & 2011 & 97,177 & 10 & $74(32-1,478)$ & $0.1(0.0-1.8)$ \\
\hline & 2012 & 98,140 & 5 & $43(8-1,321)$ & $0.1(0.0-1.6)$ \\
\hline & 2013 & 100,420 & 10 & $73(32-1,066)$ & $0.1(0.0-1.3)$ \\
\hline & 2014 & 103,481 & 7 & $77(16-934)$ & $0.1(0.0-1.2)$ \\
\hline & 2015 & 105,882 & 11 & $116(41-879)$ & $0.1(0.1-1.1)$ \\
\hline & 2016 & 107,517 & 6 & $65(25-751)$ & $0.1(0.0-0.9)$ \\
\hline \multirow[t]{6}{*}{ Toxoplasmosis during pregnancy } & 2011 & 63,102 & 18 & $252(144-485)$ & $29.3(16.7-56.4)$ \\
\hline & 2012 & 53,178 & 14 & $226(110-478)$ & $32.0(15.6-67.7)$ \\
\hline & 2013 & 53,476 & 16 & $324(171-619)$ & $44.4(23.4-84.9)$ \\
\hline & 2014 & 52,951 & 21 & $296(176-560)$ & $40.5(24.1-76.6)$ \\
\hline & 2015 & 53,900 & 21 & $450(247-803)$ & $60.3(33.0-107.5)$ \\
\hline & 2016 & 37,302 & 14 & $186(99-431)$ & $35.3(18.7-81.7)$ \\
\hline
\end{tabular}

of $\approx 8,000$ annual toxoplasmosis patients among 83 million residents of Germany offers a new assessment.

Our analysis indicates a potentially declining incidence of non-pregnancy-associated toxoplasmosis as well as toxoplasmosis during pregnancy in 2011-2016, except in 2015. This finding is in line with decreasing infections in the Netherlands (5) and France (6), as well as decreasing seroprevalence observed in Switzerland (7) and the United States (8). We hypothesize that, in France and the Netherlands, decreasing incidence is a result of improved practices in meat production, modern farming systems, increased use of frozen meat by consumers, or changes in food habits $(5,6)$. Changing diets may also play a role in the decreasing incidence in our study; vegetarianism was shown to be negatively associated with T. gondii seropositivity in Germany (11). The observed nonsignificant increase of disease incidence in 2015 might be explained by a fluctuation of T. gondii exposure because, during this time, no programs were in- troduced by public health or veterinary health services that may have affected the number of diagnoses. We are not aware of potential changes from the healthcare sector regarding medical claims policies. Disease incidence in some Germany federal states seems higher, despite lacking statistical significance. Geographic differences in raw meat consumption in Germany, such as consumption of the regional specialty food Hackepeter, were previously shown in a cross-sectional survey (18). These observations are in line with the geographic differences of toxoplasmosis incidence found in our analysis.

For congenital toxoplasmosis the mandatory disease surveillance system reported 6-20 cases of congenital toxoplasmosis each year, 2011-2016 (12). Our analysis found 43-116 cases in Germany for the same years, confirming the suspicion that the surveillance system likely has underascertainment and underreporting. Our analysis, limited to children $\leq 12$ months of age, might still underestimate the incidence of congenital 
Table 3. Estimated incidence of ocular toxoplasmosis manifestations by age group, sex, and region, Germany, 2016*

\begin{tabular}{|c|c|c|c|}
\hline Characteristic & No. patients in database & No. patients in country $(95 \% \mathrm{Cl})$ & $\begin{array}{l}\text { No. cases } / 100,000 \\
\text { population }(95 \% \mathrm{Cl})\end{array}$ \\
\hline \multicolumn{4}{|c|}{ ( } \\
\hline M & 34 & $516(346-948)$ & $1.3(0.9-2.3)$ \\
\hline $\mathrm{F}$ & 41 & 707 (494-1,389) & $1.7(1.2-3.3)$ \\
\hline \multicolumn{4}{|l|}{ Age group } \\
\hline$<1 \mathrm{y}$ & $<5$ & NA & NA \\
\hline $1-5 y$ & $<5$ & NA & NA \\
\hline $6-10 y$ & $<5$ & NA & NA \\
\hline $11-20 y$ & 5 & $70(22-329)$ & $0.9(0.3-4.1)$ \\
\hline $21-30 y$ & 9 & $160(73-432)$ & $1.6(0.7-4.3)$ \\
\hline $31-40 y$ & 9 & $157(65-421)$ & $1.5(0.6-4.1)$ \\
\hline $41-50 y$ & 11 & $178(81-430)$ & $1.6(0.7-3.7)$ \\
\hline $51-60 y$ & 22 & $354(212-653)$ & $2.7(1.6-5.1)$ \\
\hline $61-70$ y & 8 & $131(56-399)$ & $1.4(0.6-4.2)$ \\
\hline$\geq 71 \mathrm{y}$ & 5 & $94(28-779)$ & $0.8(0.2-6.4)$ \\
\hline \multicolumn{4}{|l|}{ Region } \\
\hline East & 6 & $172(58-861)$ & $1.1(0.4-5.3)$ \\
\hline West & 69 & $1,052(810-1,400)$ & $1.6(1.2-2.1)$ \\
\hline North & 20 & $415(244-819)$ & $2.0(1.2-3.9)$ \\
\hline Middle & 33 & $492(331-1,157)$ & $1.7(1.2-4.1)$ \\
\hline South & 22 & $317(197-559)$ & $1.1(0.7-1.9)$ \\
\hline Total & 75 & $1,224(941-1,931)$ & $1.5(1.1-2.3)$ \\
\hline
\end{tabular}

toxoplasmosis compared with the postulated annual total of 345 neonates in Germany, because of potential development of clinical symptoms later in life $(11,19,20)$. Another reason for an underestimation could be the lack of systematic screening of infants for congenital toxoplasmosis, which could prevent difficulties with diagnosis (21). Nevertheless, our analysis provides an improved estimation compared with the mandatory disease surveillance system.

Among non-pregnancy-associated toxoplasmosis, most patients were not further specified by disease manifestation, resulting in a large proportion of underascertainment; this phenomenon was similarly observed by Lykins et al. (16). Ocular toxoplasmosis was the non-pregnancy-associated disease manifestation with the highest incidence seen in our analysis, which is also in line with the results for the United States (16). The annual incidence in Germany of 2.0/100.000 population is roughly double the incidence reported by Lykins et al. in the United States. We would expect an even higher incidence in Germany, given the $\approx 4$ times higher seroprevalence in Germany $(49.1 \%)$ compared with the United States $(12.4 \%)(11,22)$. The large number of patients in our

\begin{tabular}{|c|c|c|c|}
\hline Characteristic & No. patients in database & No. patients in country $(95 \% \mathrm{Cl})$ & $\begin{array}{c}\text { No. cases/100,000 population } \\
(95 \% \mathrm{Cl})\end{array}$ \\
\hline \multicolumn{4}{|l|}{ Sex } \\
\hline M & 5 & $108(24-533)$ & $0.3(0.1-1.3)$ \\
\hline $\mathrm{F}$ & 5 & $92(25-778)$ & $0.2(0.1-1.9)$ \\
\hline \multicolumn{4}{|l|}{ Age group, y } \\
\hline$<1 \mathrm{y}$ & 0 & 0 & 0 \\
\hline $1-5 y$ & 0 & 0 & 0 \\
\hline $6-10 y$ & 0 & 0 & 0 \\
\hline $11-20 y$ & 0 & 0 & 0 \\
\hline $21-30 y$ & 0 & 0 & 0 \\
\hline $31-40 y$ & 0 & 0 & 0 \\
\hline $41-50 y$ & 5 & $123(29-395)$ & $1.1(0.3-3.4)$ \\
\hline $51-60 y$ & $<5$ & NA & NA \\
\hline $61-70 y$ & $<5$ & NA & NA \\
\hline$\geq 71 \mathrm{y}$ & $<5$ & NA & NA \\
\hline \multicolumn{4}{|l|}{ Region } \\
\hline East & $<5$ & NA & NA \\
\hline West & 8 & $113(46-319)$ & $0.2(0.1-0.5)$ \\
\hline North & $<5$ & NA & NA \\
\hline Middle & $<5$ & NA & NA \\
\hline South & $<5$ & NA & NA \\
\hline Total & 10 & $200(74-883)$ & $0.2(0.1-1.1)$ \\
\hline
\end{tabular}


Table 5. Estimated incidence of other unspecified toxoplasmosis manifestations by age group, sex, and region, Germany, 2016*

\begin{tabular}{|c|c|c|c|}
\hline Characteristic & No. patients in database & No. patients in country $(95 \% \mathrm{Cl})$ & $\begin{array}{c}\text { No. cases } / 100,000 \text { population } \\
(95 \% \mathrm{Cl})\end{array}$ \\
\hline \multicolumn{4}{|c|}{ 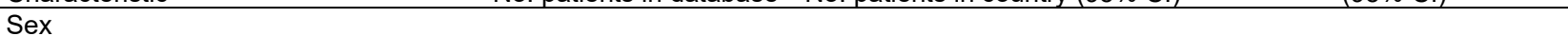 } \\
\hline M & 64 & $1,181(863-1,730)$ & $2.9(2.1-4.3)$ \\
\hline $\mathrm{F}$ & 221 & $4,039(3,384-5,048)$ & $9.7(8.1-12.1)$ \\
\hline \multicolumn{4}{|l|}{ Age group, $y$} \\
\hline$<1 y$ & 0 & 0 & 0 \\
\hline $1-5 y$ & 0 & 0 & 0 \\
\hline $6-10 y$ & $<5$ & NA & NA \\
\hline $11-20 y$ & 18 & $430(226-812)$ & $5.4(2.8-10.1)$ \\
\hline $21-30 y$ & 80 & $1,704(1,306-2,247)$ & $16.9(12.9-22.2)$ \\
\hline $31-40 y$ & 83 & $1,399(1,083-1,846)$ & $13.8(10.7-18.2)$ \\
\hline $41-50 y$ & 46 & $582(422-865)$ & $5.1(3.7-7.5)$ \\
\hline $51-60 y$ & 33 & $540(356-873)$ & $4.2(2.8-6.8)$ \\
\hline $61-70 y$ & 9 & $149(68-419)$ & $1.6(0.7-4.5)$ \\
\hline$>71$ y & 14 & $390(102-1,235)$ & $3.2(0.8-10.1)$ \\
\hline \multicolumn{4}{|l|}{ Region } \\
\hline East & 35 & $1,466(932-2,430)$ & $9.1(5.8-15.0)$ \\
\hline West & 250 & $3,754(3,292-4,314)$ & $5.7(5.0-6.5)$ \\
\hline North & 73 & $1,564(1,203-2,116)$ & $7.5(5.7-10.1)$ \\
\hline Middle & 109 & $1,896(1,413-2,773)$ & $6.7(5.0-9.7)$ \\
\hline South & 98 & $1,423(1,152-1,795)$ & $4.9(4.0-6.2)$ \\
\hline Total & 242 & $4,569(3,862-5,628)$ & $5.5(4.7-6.8)$ \\
\hline
\end{tabular}

dataset with unspecified toxoplasmosis probably contributes to this remaining underestimation compared to seroprevalence in Germany.

Our data on disease relapse also reveal a high relapse for ocular toxoplasmosis. These results are not surprising because ocular toxoplasmosis is common in immune-competent patients $(24,25)$, constituting most of the population. It can develop during childhood or adolescence even if infected neonates were born without symptoms for congenital toxoplasmosis $(19,20,26)$. An estimated $\approx 2 \%$ of T. gondii infections lead to ocular toxoplasmosis (23); this would translate into 22 patients/100,000 population annually according to the seroprevalence seen in Germany in 2008 (11). We see a lower average annual incidence of 2 patients/100,000 population in this analysis, possibly resulting from underdiagnosis and missing specification of toxoplasmosis cases. We further suspect an underestimation of incidence for cerebral as well as other types of toxoplasmo- sis, including pneumonitis and hepatitis or other organs affected, especially in immunocompromised patients; these are opportunistic infections among this group of patients and may therefore remain unrecognized.

Analysis of underlying conditions shows an association with psychiatric conditions (anxiety) and alternative diagnoses (visual loss, lymphadenopathy). Our analysis found an association with the known conditions HIV/AIDS and encephalitis, myelitis, or encephalomyelitis. However, we were unable to provide odds ratios for these conditions because of the low number of patients with HIV or encephalitis in our reference population. Directionality of conditions remains unclear from this analysis, and we were not able to confirm most associations found by Lykins et al. (16) on the basis of our dataset.

One limitation of this study is that healthcare claims are primarily intended for financial reimbursement rather than disease surveillance or clinical research.

\begin{tabular}{|c|c|c|c|}
\hline Characteristic & $\begin{array}{l}\text { No. patients identified } \\
\text { in database }\end{array}$ & No. patients in country $(95 \% \mathrm{Cl})$ & $\begin{array}{c}\text { No. cases/100,000 pregnancies } \\
(95 \% \mathrm{Cl})\end{array}$ \\
\hline \multicolumn{4}{|c|}{ (2. } \\
\hline $15-20 y$ & $<5$ & NA & NA \\
\hline $21-30 y$ & $<5$ & NA & NA \\
\hline $31-40 y$ & 10 & $134(61-351)$ & $52.7(24.1-138.1)$ \\
\hline $41-50 y$ & $<5$ & NA & NA \\
\hline \multicolumn{4}{|l|}{ Region } \\
\hline East & $<5$ & NA & NA \\
\hline West & 12 & $145(75-303)$ & $34.4(17.7-71.9)$ \\
\hline North & 5 & $78(23-238)$ & $58.2(17.2-177.9)$ \\
\hline Middle & $<5$ & NA & NA \\
\hline South & 6 & $73(27-176)$ & $37.3(13.7-89.8)$ \\
\hline Total & 14 & $186(99-431)$ & $35.3(18.7-81.7)$ \\
\hline
\end{tabular}


Table 7. Underlying conditions among patients with ocular, cerebral, or other toxoplasmosis manifestations, Germany, 20122016

\begin{tabular}{lc}
\hline Condition & Odds ratio $(95 \% \mathrm{Cl})$ \\
\hline Epilepsy & $2.1(1.3-3.3)$ \\
Visual loss, blindness, etc. & $2.7(1.7-4.3)$ \\
Anxiety & $1.5(1.2-1.8)$ \\
Thrombocytopenia & $3.2(1.7-6.1)$ \\
Lymphadenopathy & $6.0(3.9-9.3)$ \\
\hline
\end{tabular}

Therefore, the results rely on accurate coding and diagnosis and should be interpreted cautiously. Comparisons between the approaches in the United States and our data are hampered by the fundamental differences between the health systems, particularly in relation to medical claims. For similar reasons, an analysis based on toxoplasmosis-specific treatment as conducted by Lykins et al. was not possible due to different clinical guidelines. An estimate of annual toxoplasmosis incidence during pregnancy from this study $(40.3 / 100,000$ pregnancies) was restricted to women receiving toxoplasmosis-specific treatment. We can assume an unknown number of additional women need diagnostic clarification because of toxoplasmosis infection suspicion, which is likely to substantially burden the health system but is not accounted for in our incidence estimation. Although we tried to eliminate falsely diagnosed toxoplasmosis relapses by applying the criteria on patients with recurring medical claims, we need to interpret these results with caution, bearing in mind the nature of healthcare claims data and the purpose of claims. Although the estimates are adjusted for age, sex, and regional distribution, the selection of health insurance providers and the socioeconomic status of their target populations represented in this dataset may have a residual effect on these estimates that we cannot account for. Furthermore, as shown by Andersohn et al. (17), the overall death rate in the database population was slightly lower than the general population in Germany. This difference may also lead to an underestimation of toxoplasmosis illness rates in our analysis.

The incidence estimates for the different toxoplasmosis manifestations in this analysis provide a clearer picture of this disease's occurrence in Germany. The average annual number of $\approx 8,000$ toxoplasmosis patients can be regarded as high, and even more undiagnosed cases are likely. Using healthcare claims data may also help other countries with improved assessments of their toxoplasmosis burden and renew the discussion for prevention measures in European Union countries and beyond. The incidence shown and the severity of symptoms and long-term sequelae of infections justify this urgent need.

Because T. gondii is a parasite with transmission and disease aspects affecting the veterinary, human, and environmental medicine sectors, an overall prevention program needs to target different levels, following an international One Health approach (27). Screening of pregnant women is one possible method of prevention, but its cost-effectiveness, health consequences for mother and child, and effectiveness of resulting treatment are debated in light of decreasing disease incidence $(28,29)$. Although informing pregnant women of food- and animal-related risks is important, as is currently done in Germany, the incidence found in our analysis raises doubts about the effectiveness of this method. So far, insufficient evidence for effectiveness of educational efforts targeted at pregnant women has been published (30). Therefore, screening should be evaluated on the basis of the national health system structure and incidence in the country in question. Other preventive strategies currently debated include screening and implementing biosafety precautions for animal farms, as well as decontaminating meat products used for raw or undercooked consumption (31). A social cost-benefit analysis in the Netherlands has shown that freezing meat products is effective to reduce disease (32); freezing could be further implemented, especially for the production of meat products that are typically consumed raw in Germany.

\section{Acknowledgments}

We thank Sandra Beermann for her support during the initial planning of this research. We thank Katharina Alpers and Jan Walter as part of the Postgraduate Training for Applied Epidemiology team, as well as the European Programme for Intervention Epidemiology Training by the European Centre for Disease Prevention and Control for their methodological advice.

This work was supported by the Robert Koch Institute, Berlin, Germany.

InGef GmbH received compensation for J.J.'s and J.W.'s work in drafting the study protocol and data analysis from Robert Koch Institute.

\section{About the Author}

Dr. Krings is a researcher at the Robert Koch Institute in Berlin, Germany, involved in various data-for-action projects. Current research interests include hepatitis B, hepatitis $\mathrm{C}$, and HIV in intravenous drug users.

\section{References}

1. Montoya JG, Liesenfeld O. Toxoplasmosis. Lancet. 2004;363:1965-76. https:/ / doi.org/10.1016/S0140-6736 (04)16412-X 
2. Robert Koch-Institut. RKI guide: toxoplasmosis. 2018 [cited 2021 Jun 21]. https:/ / www.rki.de/DE/Content/Infekt/ EpidBull/Merkblaetter/Ratgeber_Toxoplasmose.html

3. Jones JL, Lopez A, Wilson M, Schulkin J, Gibbs R. Congenital toxoplasmosis: a review. Obstet Gynecol Surv. 2001;56: 296-305. https://doi.org/10.1097/00006254-200105000-00025

4. Pappas G, Roussos N, Falagas ME. Toxoplasmosis snapshots: global status of Toxoplasma gondii seroprevalence and implications for pregnancy and congenital toxoplasmosis. Int J Parasitol. 2009;39:1385-94. https:/ / doi.org/ 10.1016/j.ijpara.2009.04.003

5. Hofhuis A, van Pelt W, van Duynhoven YT, Nijhuis CD, Mollema L, van der Klis FR, et al. Decreased prevalence and age-specific risk factors for Toxoplasma gondii IgG antibodies in the Netherlands between 1995-1996 and 2006-2007. Epidemiol Infect. 2011;139:530-8. https:/ / doi.org/10.1017/ S0950268810001044

6. Nogareda F, Le Strat Y, Villena I, De Valk H, Goulet V. Incidence and prevalence of Toxoplasma gondii infection in women in France, 1980-2020: model-based estimation. Epidemiol Infect. 2014;142:1661-70. https://doi.org/10.1017/ S0950268813002756

7. Rudin C, Hirsch HH, Spaelti R, Schaedelin S, Klimkait T. Decline of seroprevalence and incidence of congenital toxoplasmosis despite changing prevention policy-three decades of cord-blood screening in north-western Switzerland. Pediatr Infect Dis J. 2018;37:1087-92. https://doi.org/10.1097/INF.0000000000001978

8. Jones JL, Kruszon-Moran D, Elder S, Rivera HN, Press C, Montoya JG, et al. Toxoplasma gondii infection in the United States, 2011-2014. Am J Trop Med Hyg. 2018;99:241-2

9. Torgerson PR, Mastroiacovo P. The global burden of congenital toxoplasmosis: a systematic review. Bull World Health Organ. 2013;91:501-8. https://doi.org/10.2471/BLT.12.111732

10. Rostami A, Riahi SM, Contopoulos-Ioannidis DG, Gamble HR, Fakhri Y, Shiadeh MN, et al. Acute toxoplasma infection in pregnant women worldwide: a systematic review and meta-analysis. PLoS Negl Trop Dis. 2019;13:e0007807. https://doi.org/10.1371/journal.pntd.0007807

11. Wilking H, Thamm M, Stark K, Aebischer T, Seeber F. Prevalence, incidence estimations, and risk factors of Toxoplasma gondii infection in Germany: a representative, cross-sectional, serological study. Sci Rep. 2016;6:22551. https:/ / doi.org/10.1038/srep22551

12. Robert Koch-Institut. Epidemiological yearbook of notifiable infectious diseases 2017 [in German]. Berlin; The Institute: 2018.

13. Zylka-Menhorn V. Toxoplasmosis test: arguments for screening [in German]. Dtsch Arztebl Int. 2013;110:446. https:/ / www.aerzteblatt.de/archiv/135207/ToxoplasmoseTest-Argumente-fuer-ein-Screening

14. Lange AE, Thyrian JR, Wetzka S, Flessa S, Hoffmann W, Zygmunt $\mathrm{M}$, et al. The impact of socioeconomic factors on the efficiency of voluntary toxoplasmosis screening during pregnancy: a population-based study. BMC Pregnancy Childbirth. 2016;16:197. https:/ / doi.org/10.1186/ s12884-016-0966-0

15. Bundesinstitut für Risikobewertung. Toxoplasmosis underestimated danger [in German]. 2010 [cited 2021 Jun 4]. https://www.bfr.bund.de/de/presseinformation/2010/02/ toxoplasmose_unterschaetzte_gefahr-32526.html

16. Lykins J, Wang K, Wheeler K, Clouser F, Dixon A, El Bissati K, et al. Understanding toxoplasmosis in the United States through "large data" analyses. Clin Infect Dis. 2016;63:468-75. https:// doi.org/10.1093/cid/ciw356

17. Andersohn F, Walker J. Characteristics and external validity of the German Health Risk Institute (HRI) database.
Pharmacoepidemiol Drug Saf. 2016;25:106-9. https:/ / doi.org/ 10.1002/pds.3895

18. Bremer V, Bocter N, Rehmet S, Klein G, Breuer T, Ammon A. Consumption, knowledge, and handling of raw meat: a representative cross-sectional survey in Germany, March 2001. J Food Prot. 2005;68:785-9. https:/ / doi.org/10.4315/ 0362-028X-68.4.785

19. Wallon M, Garweg JG, Abrahamowicz M, Cornu C, Vinault S, Quantin C, et al. Ophthalmic outcomes of congenital toxoplasmosis followed until adolescence. Pediatrics. 2014;133:e601-8. https:/ / doi.org/10.1542/peds.2013-2153

20. Faucher B, Garcia-Meric P, Franck J, Minodier P, Francois P, Gonnet $\mathrm{S}$, et al. Long-term ocular outcome in congenital toxoplasmosis: a prospective cohort of treated children. J Infect. 2012;64:104-9. https:// doi.org/10.1016/j.jinf.2011.10.008

21. Wallon M, Peyron F. Congenital toxoplasmosis: a plea for a neglected disease. Pathogens. 2018;7:25. https://doi.org/ 10.3390/pathogens7010025

22. Jones JL, Kruszon-Moran D, Rivera HN, Price C, Wilkins PP. Toxoplasma gondii seroprevalence in the United States 2009-2010 and comparison with the past two decades. Am J Trop Med Hyg. 2014;90:1135-9. https:// doi.org/10.4269/ ajtmh.14-0013

23. Jones JL, Holland GN. Annual burden of ocular toxoplasmosis in the US. Am J Trop Med Hyg. 2010;82:464-5. https:/ / doi.org/10.4269/aitmh.2010.09-0664

24. Pleyer U, Gross U, Schlüter D, Wilking H, Seeber F. Toxoplasmosis in Germany. Dtsch Arztebl Int. 2019;116:435-44.

25. Maenz M, Schlüter D, Liesenfeld O, Schares G, Gross U, Pleyer U. Ocular toxoplasmosis past, present, and new aspects of an old disease. Prog Retin Eye Res. 2014;39:77-106. https:// doi.org/10.1016/j.preteyeres.2013.12.005

26. Wallon M, Kodjikian L, Binquet C, Garweg J, Fleury J, Quantin C, et al. Long-term ocular prognosis in 327 children with congenital toxoplasmosis. Pediatrics. 2004;113:1567-72. https://doi.org/10.1542/peds.113.6.1567

27. Aguirre AA, Longcore T, Barbieri M, Dabritz H, Hill D, Klein PN, et al. The One Health approach to toxoplasmosis: epidemiology, control, and prevention strategies. EcoHealth. 2019;16:378-90. https://doi.org/10.1007/s10393-019-01405-7

28. Khoshnood B, De Vigan C, Goffinet F, Leroy V. Prenatal screening and diagnosis of congenital toxoplasmosis: a review of safety issues and psychological consequences for women who undergo screening. Prenat Diagn. 2007;27:395403. https:// doi.org/10.1002/pd.1715

29. Picone O, Fuchs F, Benoist G, Binquet C, Kieffer F, Wallon M, et al. Toxoplasmosis screening during pregnancy in France: opinion of an expert panel for the CNGOF. J Gynecol Obstet Hum Reprod. 2020;49:101814. https://doi.org/10.1016/ j.jogoh.2020.101814

30. Di Mario S, Basevi V, Gagliotti C, Spettoli D, Gori G, D'Amico R, et al. Prenatal education for congenital toxoplasmosis. Cochrane Database Syst Rev. 2015; (10):CD006171.

31. Opsteegh M, Kortbeek TM, Havelaar AH, van der Giessen JW. Intervention strategies to reduce human Toxoplasma gondii disease burden. Clin Infect Dis. 2015;60:101-7. https://doi.org/10.1093/cid/ciu721

32. Suijkerbuijk AWM, Over EAB, Opsteegh M, Deng H, Gils PFV, Bonačić Marinović AA, et al. A social costbenefit analysis of two One Health interventions to prevent toxoplasmosis. PLoS One. 2019;14:e0216615. https:// doi.org/ 10.1371/journal.pone.0216615

Address for correspondence: Amrei Krings, Robert Koch Institute, Seestraße 10, 13353 Berlin, Germany; email: kringsa@rki.de 